CERN-PPE/95-134

17 August 1995

\title{
First Observation of a Particle-Antiparticle Asymmetry in the Decay of Neutral Kaons into $\pi^{0} \pi^{0}$
}

\begin{abstract}
CPLEAR Collaboration
R. Adler ${ }^{2}$, T. Alhalel ${ }^{11}$, A. Angelopoulos ${ }^{1}$, A. Apostolakis ${ }^{1}$, E. Aslanides ${ }^{11}$, G. Backenstoss ${ }^{2}$, C.P. Bee ${ }^{9}$, O. Behnke ${ }^{17}$, A. Benelli ${ }^{9}$, J. Bennet ${ }^{9}$, V. Bertin ${ }^{11}$, J.K. Bienlein ${ }^{17}$, F. Blanc ${ }^{7,13}$, P. Bloch ${ }^{4}$, C. Bula ${ }^{13}$, P. Carlson ${ }^{15}$, M. Carroll ${ }^{9}$, J. Carvalho ${ }^{5}$, E. Cawley ${ }^{9}$, S. Charalambous ${ }^{16}$, M. Chardalas ${ }^{16}$, G. Chardin ${ }^{14}$,
\end{abstract} M.B. Chertok ${ }^{3}$, A. Cody ${ }^{9}$, M. Danielsson ${ }^{15}$, S. Dedoussis ${ }^{16}$, M. Dejardin ${ }^{14}$, J. Derre ${ }^{14}$, M. Dodgson ${ }^{9}$, J. Duclos ${ }^{14}$, A. Ealet ${ }^{11}$, B. Eckart ${ }^{2}$, C. Eleftheriadis ${ }^{16}$, I. Evangelou ${ }^{8}$, L. Faravel $^{7}$, P. Fassnacht ${ }^{11}$, J.L. Faure ${ }^{14}$, C. Felder ${ }^{2}$, R. Ferreira-Marques ${ }^{5}$, W. Fetscher ${ }^{17}$, M. Fidecaro ${ }^{4}$, A. Filipčič ${ }^{10}$, D. Francis ${ }^{3}$, J. Fry ${ }^{9}$, C. Fuglesang ${ }^{15}$, E. Gabathuler ${ }^{9}$, R. Gamet ${ }^{9}$, D. Garreta ${ }^{14}$, T. Geralis ${ }^{13}$, H.-J. Gerber ${ }^{17}$, A. Go $^{3,15}$, P. Gumplinger ${ }^{17}$, C. Guyot ${ }^{14}$, P.F. Harrison ${ }^{9}$, A. Haselden ${ }^{9}$, P.J. Hayman ${ }^{9}$, F. Henry-Couannier ${ }^{11}$, W.G. Heyes ${ }^{4}$, R.W. Hollander ${ }^{6}$, E. Hubert ${ }^{11}$, K. Jansson ${ }^{15}$, H.U. Johner ${ }^{7}$, K. Jon-And ${ }^{15}$, P.R. Kettle ${ }^{13}$, C. Kochowski ${ }^{14}$, P. Kokkas ${ }^{2}$, R. Kreuger ${ }^{6}$,

T. Lawry ${ }^{3}$, R. Le Gac ${ }^{11}$, F. Leimgruber ${ }^{2}$, A. Liolios ${ }^{16}$, E. Machado ${ }^{5}$, P. Maley ${ }^{9}$, I. Mandić ${ }^{10}$, N. Manthos ${ }^{8}$, G. Marel ${ }^{14}$, M. Mikuž ${ }^{10}$, J. Miller ${ }^{3}$, F. Montanet ${ }^{11}$, T. Nakada ${ }^{13}$, A. Onofre ${ }^{5}$, B. Pagels ${ }^{17}$, I. Papadopoulos ${ }^{16}$, P. Pavlopoulos ${ }^{2}$, F. Pelucchi ${ }^{11}$, J. Pinto da Cunha ${ }^{5}$, A. Policarpo ${ }^{5}$, G. Polivka ${ }^{2}$, H. Postma ${ }^{6}$, R. Rickenbach ${ }^{2}$, B.L. Roberts ${ }^{3}$, E. Rozaki ${ }^{1}$, T. Ruf ${ }^{4}$, L. Sacks ${ }^{9}$, L. Sakeliou ${ }^{1}$, P. Sanders ${ }^{9}$, C. Santoni ${ }^{2}$, K. Sarigiannis ${ }^{1}$, M. Schäfer ${ }^{17}$, L.A. Schaller ${ }^{7}$, T. Schietinger ${ }^{2}$, A. Schopper ${ }^{4}$, P. Schune ${ }^{14}$, A. Soares ${ }^{14}$, L. Tauscher ${ }^{2}$, C. Thibault ${ }^{12}$, F. Touchard ${ }^{11}$, C. Touramanis ${ }^{4}$, F. Triantis ${ }^{8}$, D.A. Tröster ${ }^{2}$, E. Van Beveren ${ }^{5}$, C.W.E. Van Eijk ${ }^{6}$, G. Varner ${ }^{3}$, S. Vlachos ${ }^{2}$, P. Weber ${ }^{17}$, O. Wigger ${ }^{13}$, C. Witzig ${ }^{17}$, M. Wolter ${ }^{17}$, C. Yeche ${ }^{14}$, D. Zavrtanik ${ }^{10}$ and D. Zimmerman ${ }^{3}$.

\begin{abstract}
$\mathrm{CP}$ violation has been observed as a time-dependent rate asymmetry between the decays $\overline{\mathrm{K}}^{0} \rightarrow \pi^{0} \pi^{0}$ and $\mathrm{K}^{0} \rightarrow \pi^{0} \pi^{0}$, where the neutral kaons are produced with definite and individually known strangeness in $\overline{\mathrm{p}} \mathrm{p} \rightarrow \overline{\mathrm{K}}^{0} \mathrm{~K}^{+} \pi^{-}$or $\overline{\mathrm{p}} \mathrm{p} \rightarrow \mathrm{K}^{0} \mathrm{~K}^{-} \pi^{+}$. A special technique for the data analysis has been developed. The values obtained for $\phi_{00}$ and $\left|\eta_{00}\right|$ are in agreement with those of previous measurements of CP violation.
\end{abstract}


We describe an experiment in which neutral kaons with definite and, event by event, unambiguously known strangeness have been produced and observed to decay into a pair of neutral pions. Since CP is not conserved, the time evolutions of the appearances of the neutral pions differ for $\overline{\mathrm{K}}^{0}$ and $\mathrm{K}^{0}$ parents. The corresponding eigentime-dependent rate asymmetry

$$
A_{00}(\tau) \equiv \frac{R\left(\overline{\mathrm{K}}^{0} \rightarrow \pi^{0} \pi^{0}\right)(\tau)-R\left(\mathrm{~K}^{0} \rightarrow \pi^{0} \pi^{0}\right)(\tau)}{R\left(\overline{\mathrm{K}}^{0} \rightarrow \pi^{0} \pi^{0}\right)(\tau)+R\left(\mathrm{~K}^{0} \rightarrow \pi^{0} \pi^{0}\right)(\tau)},
$$

which exhibits values of order 0.5 , has been directly observed for the first time. Since $R\left(\overline{\mathrm{K}}^{0} \rightarrow \pi^{0} \pi^{0}\right)(\tau)$ and $R\left(\mathrm{~K}^{0} \rightarrow \pi^{0} \pi^{0}\right)(\tau)$ correspond to $\mathrm{CP}$ conjugate processes, any difference between the two is a sign of $\mathrm{CP}$ violation, independent of phenomenological descriptions.

Results based on the appropriate asymmetry concerning the decay of neutral kaons into pairs of charged pions have been reported previously [1][2].

Classical interference experiments on $\mathrm{CP}$ violation in the past [3], studying the neutral decay channel, differ from the present experiment in that they have been made with neutral kaons whose strangeness has not been individually determined at $\tau=0$.

In the CPLEAR experiment the neutral kaons are produced in the annihilations $\overline{\mathrm{p}} \mathrm{p} \rightarrow \overline{\mathrm{K}}^{0} \mathrm{~K}^{+} \pi^{-}$and $\overline{\mathrm{p}} \mathrm{p} \rightarrow \mathrm{K}^{0} \mathrm{~K}^{-} \pi^{+}$. The strangeness conservation in the annihilation process affirms whether the neutral kaon is created as a $\overline{\mathrm{K}}^{0}$ or as a $\mathrm{K}^{0}$ depending on the sign of the charge of the simultaneously produced charged kaon.

In order to measure $A_{00}(\tau)$, a certain precision in locating the neutral kaon decay point is required. Due to the steeply falling decay-time distribution, even a small fraction of early kaon decays with poorly reconstructed decay positions may contribute false events to the later decay-time region and disturb the asymmetry measurement. Since the particles at the kaon decay point are all invisible, we determine its position by a full reconstruction of the neutral particle cascade $\overline{\mathrm{K}}^{0}\left(\mathrm{~K}^{0}\right) \rightarrow 2 \pi^{0} \rightarrow 4 \gamma$. Regular methods of geometrical and kinematical constraint fits fail to reconstruct a sizable fraction of events with the required decay-time accuracy, due to the nonlinearity of the constraints, and to the fact that certain kinematical configurations inherently lack the mathematical information. We will describe the principle of a technique for deciding whether a given event would allow for a sufficiently precise and unambiguous decay point reconstruction, and thus be included

\footnotetext{
1) University of Athens, GR-10680 Athens, Greece

2) University of Basle, CH-4056 Basle, Switzerland

3) Boston University, Boston, MA 02215, USA

4) CERN, CH-1211 Geneva 23, Switzerland

5) LIP and University of Coimbra, P-3000 Coimbra, Portugal

6) Delft University of Technology, 2629 JB Delft, Netherlands

7) University of Fribourg, CH-1700 Fribourg, Switzerland

8) University of Ioannina, GR-45110 Ioannina, Greece

9) University of Liverpool, Liverpool L69 3BX, UK

10) J. Stefan Inst. and Phys. Dep., University of Ljubljana, SI-61111 Ljubljana, Slovenia

11) CPPM, IN2P3-CNRS et Université d'Aix-Marseille II, F-13288 Marseille, France

12) CSNSM, IN2P3-CNRS, F-91405 Orsay, France

13) Paul-Scherrer-Institut(PSI), CH-5232 Villigen, Switzerland

14) CEA, DSM/DAPNIA, CE-Saclay, F-91191 Gif-sur-Yvette, France

15) Royal Institute of Technology, S-10405 Stockholm, Sweden

16) University of Thessaloniki, GR-54006 Thessaloniki, Greece

17) ETH-IPP Zürich, CH-8093 Zürich, Switzerland
} 
in the final sample. Having applied this technique, we observed the $\mathrm{CP}$ violation rate asymmetry and extracted the parameters $\phi_{00}$ and $\left|\eta_{00}\right|$. The results are in agreement with those of previous measurements of CP violation.

The rate asymmetry method of CPLEAR and the special event selection and reconstruction criteria we have applied, result in a value of $\phi_{00}$ with a competitively small systematic error. The present accuracy is limited by the number of events available.

\section{$2 \quad$ Experimental Method}

Antiprotons from the Low Energy Antiproton Ring LEAR at CERN, with a momentum of $200 \mathrm{MeV} / \mathrm{c}$, annihilate at rest in hydrogen gas (16 bar) at the center of the CPLEAR detector [4]. In a fraction of $\approx 4 \times 10^{-3}$ of the annihilations the reaction $\overline{\mathrm{p}} \mathrm{p} \rightarrow \mathrm{K}+\mathrm{K}+\pi$ occurs, where one of the kaons is charged, and one is neutral. In the CPLEAR detector the tracking is performed with two layers of proportional chambers, six layers of drift chambers and two layers of streamer tubes. The charged particle identification $\left(\mathrm{K}^{ \pm} \pi^{\mp}\right)$ is achieved with a sandwich of scintillator-Čerenkov-scintillator counters. The electromagnetic showers are detected in a lead/gas sampling calorimeter using 17280 tubes, each with one wire, working in a limited streamer mode. It consists of three segments, each of $120^{\circ}$, and of 18 layers in depth. Each layer of tubes is sandwiched between two layers of pick-up strips $\left( \pm 30^{\circ}\right.$ with respect to the tubes $)$. The whole apparatus, including the electromagnetic calorimeter, is located inside a solenoidal magnet (radius: $1 \mathrm{~m}$; field: $0.44 \mathrm{~T}$ ). For a detailed description, see Ref. [4]. Some detector components and the visible tracks of an event containing the cascade $\mathrm{K}^{0} \rightarrow 2 \pi^{0} \rightarrow 4 \gamma$, together with the reconstructed invisible paths of the neutrals, are shown in Fig. (1).

The neutral kaon direction of flight and its momentum can be predicted, using fourmomentum conservation, from the measurement of the trajectories of the charged $\mathrm{K}$ and $\pi$ in the tracking devices. The determination of the neutral kaon's decay point along its direction of flight requires the identification of the four photons in the electromagnetic calorimeter. The conversion point of a photon in the calorimeter is measured with a pre-

cision of $4.5 \mathrm{~mm}$ in space, the photon energy with a precision of $\sigma_{E} / E=15 \% / \sqrt{E[\mathrm{GeV}]}$, and the photon direction is reconstructed with a precision of $250 \mathrm{mrad}$ for $100 \mathrm{MeV}$ photons. It is the precise measurement of the photon conversion points that contributes most of the additional information required for finding the decay length of the event, whereas the measured energy and shower directions do little in the reconstruction [5]. The final decay-time resolution is equally determined by the precision of the $\mathrm{K}^{0}$ four-momentum as by the precision of the photon impact point positions.

\section{$3 \quad$ Event Selection and Reconstruction}

Here we report on data collected from 1992 to 1994 . During this period, the trigger selected, among other classes of events, two-track events having at least one kaon candidate and at least five clusters of deposited charge detected in the calorimeter. Clusters detected in the electromagnetic calorimeter can initially be due to either neutral or charged particles. Their online selection is described elsewhere [4].

In the offline analysis, the identification of the charged kaon and pion is performed with more refined information from the charged particle momentum, the energy loss in the inner scintillator and the time of flight measurement. The event hypothesis $\bar{p} p \rightarrow$ $\overline{\mathrm{K}}^{0}\left(\mathrm{~K}^{0}\right) \mathrm{K}^{ \pm} \pi^{\mp}$ is then tested with a constraint fit requiring the missing mass of the $\mathrm{K}^{ \pm} \pi^{\mp}$ pair to agree with the mass of the neutral kaon. The decay $\overline{\mathrm{K}}^{0}\left(\mathrm{~K}^{0}\right) \rightarrow 2 \pi^{0} \rightarrow 4 \gamma$ is selected 
by requiring the detection of exactly four electromagnetic showers in the calorimeter, unassociated with any charged particle's track.

The reconstruction of the showers is provided by the shower-pattern recognition algorithm described in Ref. [4]. The efficiency for reconstructing one photon pointing to the calorimeter is $95 \%$ for photons of $150 \mathrm{MeV}$ or more and drops linearly to $50 \%$ for photons of $50 \mathrm{MeV}$. We note that on average the least energetic photon from the decay of $\overline{\mathrm{K}}^{0}\left(\mathrm{~K}^{0}\right) \rightarrow 2 \pi^{0} \rightarrow 4 \gamma$ carries only $60 \mathrm{MeV}$. The efficiency in reconstructing all four photons is $20 \%$. Taking into account the $40 \%$ geometrical $4 \gamma$-acceptance of the calorimeter, the total efficiency in detecting the four photons amounts to $8 \%$.

To determine the neutral kaon decay vertex, a $6 c$-fit is introduced and three additional criteria, described in the following section, are applied to the selected events. These criteria, which are the special features of the present analysis, were optimized to achieve a decay-time resolution and a background rejection for obtaining the best sensitivity on the measured parameters. The final data sample consists of 1.4 million events.

\section{Technique for the Reconstruction of the Neutral Kaon Decay Vertex}

The asymmetry, Eq. (1), which provides the CP violation parameters, is

$$
A_{00}(\tau)=2 \operatorname{Re}(\epsilon)-\frac{2\left|\eta_{00}\right| e^{-\frac{1}{2}\left(1 / \tau_{L}-1 / \tau_{S}\right) \tau} \cos \left(\Delta m \tau-\phi_{00}\right)}{1+\left|\eta_{00}\right|^{2} e^{-\left(1 / \tau_{L}-1 / \tau_{S}\right) \tau}}
$$

as derived from the time dependence of the $\overline{\mathrm{K}}^{0}\left(\mathrm{~K}^{0}\right)$ decay rates into $\pi^{0} \pi^{0}$

$$
\begin{aligned}
R\left(\stackrel{(-)}{\mathrm{K}} \rightarrow \pi^{0} \pi^{0}\right)(\tau)= & \Gamma_{S}^{\pi^{0} \pi^{0}}[1 \mp 2 \operatorname{Re}(\epsilon)]\left(e^{-\tau / \tau_{S}}+\left|\eta_{\mathrm{OO}}\right|^{2} e^{-\tau / \tau_{L}}\right. \\
& \left. \pm 2\left|\eta_{\mathrm{OO}}\right| e^{-\frac{1}{2}\left(1 / \tau_{S}+1 / \tau_{L}\right) \tau} \cos \left(\Delta m \tau-\phi_{\mathrm{OO}}\right)\right),
\end{aligned}
$$

where $\tau$ denotes the decay eigentime of the neutral kaon, $\tau_{S}\left(\tau_{L}\right)$ is the $\mathrm{K}_{\mathrm{S}}^{0}\left(\mathrm{~K}_{\mathrm{L}}^{0}\right)$ mean life, $\Delta m=m_{L}-m_{S}$ their mass difference, $\eta_{00}$ is the $\mathrm{CP}$ violation parameter, $\phi_{00}$ its phase, $\epsilon$ the $C P$ violation $\overline{\mathrm{K}}^{0}-\mathrm{K}^{0}$ mixing parameter and $\Gamma_{S}^{\pi^{0}} \pi^{0}$ the $\mathrm{K}_{\mathrm{S}}^{0} \rightarrow \pi^{0} \pi^{0}$ decay width.

The accuracy of the reconstruction of the neutral kaon decay-time required to measure $A_{00}(\tau)$ is illustrated in Fig. (2a), where the expected experimental asymmetry is displayed for three time resolutions. The curve labelled ideal (infinitely good resolution) shows $A_{00}(\tau)$. The curve labelled regular is the result of an analysis following the usual procedure of constraint fits to the data, where the long tails of the decay-time resolution function, Fig. (2b), wash out the asymmetry to a great extent.

We have therefore been forced to enlarge the procedure by making use of some specific properties of well reconstructed events. In the following we describe the three additional selection criteria mentioned above. The curves labelled this technique in Fig. (2) show the achievement of our analysis. A detailed account is given in Ref. [6].

Before applying the criteria, the neutral kaon decay-time is reconstructed by a 6c-fit, i.e. by minimizing the function $\chi^{2}(\vec{m}, \tau, \vec{\lambda})$ with respect to $\vec{m}, \tau$ and $\vec{\lambda}$ (regular analysis). The vector $\vec{m}$ represents the 21 measured quantities, namely: the momenta of the primary tracks, the $\overline{\mathrm{K}}^{0}\left(\mathrm{~K}^{0}\right)$ production vertex, the photon impact points (only the $\varphi$ and $z$ components are used, whilst the $r$ component is fixed at the radius of the shower apex during the fit) and the photon energies. $\tau$ is the unknown kaon decay-time. The vector $\vec{\lambda}$ represents Lagrange multipliers corresponding to the seven constraints. These are: energy and momentum conservation (4c), the equality of the missing mass at the 
primary vertex with the $\mathrm{K}^{0}$ mass $(1 \mathrm{c})$, and the $\gamma \gamma$ invariant masses of the two $\gamma \gamma$ pairs to be equal to the $\pi^{0}$ mass $(2 \mathrm{c})$. For each event we require the probability from the $6 \mathrm{c}$-fit to be greater than $10 \%$.

In order to apply the $6 \mathrm{c}$-fit to data, we have to know the correct association of the four photons to their parent $\pi^{0} \mathrm{~s}$. In a first step, we calculate $\chi_{\min }^{2}$ for each of the three possible photon pairings, and we choose that pairing which leads to the lowest value of $\chi_{\min }^{2}$. As can be shown from simulation, the correct pairing is found only in $90 \%$ of the cases. The first additional criterion serves to uncover events where the above reconstruction selected the wrong pairing. We note the relation between the mass of the neutral kaon and the invariant masses of all the pair combinations of the four photons into which it decays:

$$
m_{K^{0}}^{2}=\sum_{i>j}^{4} m_{i j}^{2} .
$$

We see that the sum $S=\sum_{k l} m_{k l}^{2}$, where the indices $(k l)$ denote those four possible photon pairings which have not originated from a $\pi^{0}$, takes the constant value $S=m_{K^{0}}^{2}-2 m_{\pi^{0}}^{2}$. The six $m_{i j}$ have thus three degrees of freedom. A simulation study has shown that the biggest of the $m_{k l}$ strongly tends to higher values when the event has been reconstructed with the wrong pairing, as discussed in Ref. [7]. This tendency is even stronger for $m_{a}$, the (normalized and absolute) value of the most significant component of a four-dimensional principal component analysis [8] performed with the $m_{k l}$. Figure (3) demonstrates that the condition $m_{a}<0.7$ excludes all events reconstructed with a wrong pairing from the test sample.

The second additional criterion serves to identify events which have a high probability of populating the tails in Fig. (2b) [6]. It is derived from a study of the shape of the $\chi^{2}$ function. For each event a probability density $w(\tau) \sim \exp \left(-\chi^{2} / 2\right)$ is calculated for a set of fixed values of $\tau$ ranging from $-20 \tau_{s}$ to $+50 \tau_{s}$. The probability that the kinematical configuration characterized by $\tau$ represents the measured quantities of the event is expressed by $w(\tau) d \tau$, and is plotted in Fig. (4) for four genuine events, generated by Monte Carlo simulation. The curves of the sub-figures (4a) and (4b) favour a small range of $\tau$-values and therefore lead to an accurate knowledge of the decay-time, whereas the curves of $(4 \mathrm{c})$ and $(4 \mathrm{~d})$ allow for a wide range of $\tau$-values and thus leave the decay-time widely undetermined. The examples also demonstrate the existence of events with more than one local maximum in the $w(\tau)$-function. Such events are not necessarily those with a poor decay-time resolution, as example (4b) illustrates, where the two $w(\tau)$-maxima are close together. One further recognizes from $(4 \mathrm{~d})$ that the shape of the $w(\tau)$-function can be very asymmetric, which reflects the nonlinearity of the constraint equations. These observations point out the need for a reliable calculation of the decay-time error. As a quantitative measure of the range of $\tau$-values of one event, we use

$$
\sigma_{\tau}^{2}=\int w(\tau)(\tau-\bar{\tau})^{2} d \tau
$$

where $\bar{\tau}=\int w(\tau) \tau d \tau$. In the following, we retain only those events with $\sigma_{\tau}<1.0 \tau_{S}$.

The third additional criterion serves to decide whether the measured shower directions agree with the four photons to have originated from the $\mathrm{K}^{0}$ decay point as determined 
by the $6 c$-fit. From the observed shower directions and the known kaon line of flight, we determine an independent decay position for the neutral kaon and its error, $\sigma_{\tau}^{s h}$, expressed as a time interval. We require that this position corresponds to a decay-time not shorter than $\tau-\sigma_{\tau}^{s h}$, where $\tau$ is the decay-time as provided by the $6 c$-fit.

To determine the resolution of the decay-time $\tau$, a detailed Monte Carlo simulation is performed. By applying the three discussed criteria to the simulated data, $59 \%$ of all $\overline{\mathrm{K}}^{0}\left(\mathrm{~K}^{0}\right) \rightarrow \pi^{0} \pi^{0}$ events are rejected as being not reconstructible with sufficient accuracy. Figure (2b) displays the obtained decay-time resolution function, which has a width of $0.7 \tau_{S}(\mathrm{FWHM})$.

\section{$5 \quad$ Results}

The $\overline{\mathrm{K}}^{0}\left(\mathrm{~K}^{0}\right)$ decay-time distribution obtained after applying the full reconstruction technique to the data is shown in Fig. (5).

Possible sources of background are other neutral kaon decays, for example $\mathrm{K}_{\mathrm{L}}^{0} \rightarrow$ $\pi^{0} \pi^{0} \pi^{0}$, and the annihilations $\overline{\mathrm{p}} \mathrm{p} \rightarrow \overline{\mathrm{K}}^{0}\left(\mathrm{~K}^{0}\right) \mathrm{K}^{ \pm} \pi^{\mp}+\pi^{0}$ and $\overline{\mathrm{p}} \mathrm{p} \rightarrow \mathrm{K}^{+} \mathrm{K}^{-}+n \cdot \pi^{0}(n \geq 0)$, which were simulated according to their branching ratios. The generated Monte Carlo events pass a code, which simulates the complete set of the CPLEAR trigger conditions, followed by the same selection and reconstruction criteria as applied to data. The contributions of background from $\overline{\mathrm{p}} \mathrm{p} \rightarrow \pi^{+} \pi^{-}+n \cdot \pi^{0}(n \geq 0)$ annihilations (pionic annihilation background) is directly determined from the data by studying the energy loss distribution of the charged particles in the inner scintillators. Table (1) summarizes the significant contributions of background to the final data sample in the measured decay-time interval $[0,20] \tau_{S}$. In the shorter decay-time region $\left(<8 \tau_{S}\right)$ the pionic annihilation background is dominant, whereas for longer decay-times the neutral kaon decay $\mathrm{K}_{\mathrm{L}}^{0} \rightarrow \pi^{0} \pi^{0} \pi^{0}$ becomes the main background. In total, the background amounts to less than $1 \%$ and does not exceed $2.5 \%$ in the interference region between $3 \tau_{S}$ and $8 \tau_{S}$.

The decay curve obtained from data agrees well with the one obtained from the simulation, as can be seen from Fig. (5). The distribution of Fig. (5) has been fitted with an exponential decay rate taking into account the background and the (slowly varying) acceptance for the signal events, and leaving the $\mathrm{K}_{\mathrm{S}}^{0}$ lifetime and the width of the resolution function as free parameters. The resulting width of the resolution function is increased by only $5 \%$ as compared to the simulation, and the value obtained for $\tau_{S}$ is in agreement with the world average value [9].

The measured decay rate asymmetry is shown in Fig. (6). One recognizes the oscillation of the $\mathrm{CP}$ violation interference, which is diluted by the decay-time resolution and additionally at large decay-times by the $\mathrm{K}_{\mathrm{L}}^{0} \rightarrow \pi^{0} \pi^{0} \pi^{0}$ background.

\subsection{Determination of $\phi_{00}$}

The CP violation parameters are obtained from the data by comparing the measured time-dependent decay rates of $\overline{\mathrm{K}}^{0}$ and $\mathrm{K}^{0}$ through the asymmetry given by Eq. (2). The measured decay rates, however, differ from the theoretical ones of Eq. (3), due to the finite $\overline{\mathrm{K}}^{0}\left(\mathrm{~K}^{0}\right)$ decay-time resolution, the residual background and acceptances. The offset of the measured asymmetry shown in Fig. (6) compared to the theoretical asymmetry shown in Fig. (2a) is due to the different efficiencies for identifying $\bar{K}^{0}$ and $K^{0}$ in the experiment. The ratio $\xi$ of these two efficiencies deviates from unity, since the charged particles used to determine the strangeness of the neutral kaon, interact differently with the detector material, depending on their charge and momentum. As a consequence, $\xi$ is a function 


\begin{tabular}{|lc|}
\hline \hline Background Channel & Contribution [\%] \\
\hline \hline $\mathrm{K}_{\mathrm{L}}^{0} \rightarrow \pi^{0} \pi^{0} \pi^{0}$ & $0.194 \pm 0.005$ \\
$\overline{\mathrm{p}} \mathrm{p} \rightarrow \overline{\mathrm{K}}^{0}\left(\mathrm{~K}^{0}\right) \mathrm{K}^{ \pm} \pi^{\mp}+\pi^{0}$ & $0.12 \pm 0.04$ \\
$\overline{\mathrm{p}} \mathrm{p} \rightarrow \mathrm{K}^{+} \mathrm{K}^{-}+n \cdot \pi^{0}(n \geq 0)$ & $0.12 \pm 0.05$ \\
$\overline{\mathrm{p}} \mathrm{p} \rightarrow \pi^{+} \pi^{-}+n \cdot \pi^{0}(n \geq 0)$ & $0.224 \pm 0.050$ \\
\hline \hline
\end{tabular}

Table 1: Background contributions to the final data sample in the measured decay-time interval $[0,20] \tau_{S}$. The quoted errors are statistical.

of the kinematical variables of the primary particles. On the other hand, for a given set of these kinematical variables, $\xi$ is independent of the decay length of the neutral kaon, since $\overline{\mathrm{K}}^{0}$ and $\mathrm{K}^{0}$ decay into the same final state $(4 \gamma)$. Hence each data sample of a given $\overline{\mathrm{K}}^{0}\left(\mathrm{~K}^{0}\right)$ momentum represents a subset of the phase space of the primary particles, which can be characterized by a single value of $\xi$ independent of the $\overline{\mathrm{K}}^{0}\left(\mathrm{~K}^{0}\right)$ decay-time. We note that adding two data samples with different momenta of the neutral kaon could introduce a decay-time dependent $\xi$ value, since the decay-time acceptance varies with the $\overline{\mathrm{K}}^{0}\left(\mathrm{~K}^{0}\right)$ momentum, for example due to the finite detector volume. To avoid this effect we divided the full data set into subsamples as a function of the momentum of the neutral kaon, in such a way that the influence from a varying $\xi$ and a varying decay-time acceptance within each subsample becomes negligible. We remark that owing to the theoretical asymmetry Eq. (2) having a constant term of $2 \operatorname{Re}(\epsilon)$, the total offset of the measured asymmetry is $(\alpha-1) / 2$, where $\alpha=\xi[1+4 \operatorname{Re}(\epsilon)]$.

The value of $\phi_{00}$ extracted from a global likelihood fit to the asymmetries corresponding to these different data samples, taking into account the finite decay-time resolution, the remaining background contributions and regeneration, is

$$
\phi_{00}=50.8^{\circ} \pm 7.1^{\circ} \text { (stat.) }
$$

The magnitude of $\eta_{00}$ and the $\alpha$-values of the $\overline{\mathrm{K}}^{0}\left(\mathrm{~K}^{0}\right)$ momentum subsamples are free parameters in the fit. We obtain $\left|\eta_{00}\right|=(2.49 \pm 0.40) 10^{-3}$ (stat.). The $\alpha$-values vary to within $5 \%$ and have a weighted average of $\langle\alpha\rangle=1.1570 \pm 0.0022$ (stat.). The solid line in Fig. (6) is calculated from the result of the fit. These values of $\phi_{00}$ and $\left|\eta_{00}\right|$ are in agreement with the PDG values [9].

\subsection{Systematic Uncertainties}

The following contributions to the systematic uncertainties in the measurement of $\phi_{00}$ are considered:

- The resolution in reconstructing the neutral kaon decay point.

- Photons from secondary interactions.

- The residual background as listed in Table (1).

- The different efficiencies in identifying $\overline{\mathrm{K}}^{0}$ and $\mathrm{K}^{0}$.

- Regeneration effects introduced by the interaction of $\overline{\mathrm{K}}^{0}$ and $\mathrm{K}^{0}$ with the detector material.

- Uncertainties on $\Delta m$ and $\tau_{S}$, which enter Eq. (2).

To estimate systematic effects due to the finite decay-time resolution, the neutral kaon decay distribution obtained from data has been fitted with the expected decay rates, 


\begin{tabular}{|lc|}
\hline \hline Source & $\Delta \phi_{00}$ \\
\hline \hline$\overline{\mathrm{K}}^{0}\left(\mathrm{~K}^{0}\right)$ decay-time resolution & $0.6^{0}$ \\
Photons from secondary interactions & $1.0^{0}$ \\
Background $\mathrm{K}_{\mathrm{L}}^{0} \rightarrow 3 \pi^{0}$ & $<0.1^{0}$ \\
Background $\overline{\mathrm{p}} \mathrm{p} \rightarrow \overline{\mathrm{K}}^{0}\left(\mathrm{~K}^{0}\right) \mathrm{K}^{ \pm} \pi^{\mp}+\pi^{0}$ & $<0.1^{0}$ \\
Background $\overline{\mathrm{p}} \mathrm{p} \rightarrow \mathrm{K}^{+} \mathrm{K}^{-}+n \cdot \pi^{0}(n \geq 0)$ & $<0.1^{0}$ \\
Background $\overline{\mathrm{p}} \mathrm{p} \rightarrow \pi^{+} \pi^{-}+n \cdot \pi^{0}(n \geq 0)$ & $0.5^{0}$ \\
$\overline{\mathrm{K}}^{0}\left(\mathrm{~K}^{0}\right)$ identification efficiencies & $0.5^{0}$ \\
$\mathrm{Coherent}$ regeneration & $0.6^{0}$ \\
\hline Total & $1.5^{0}$ \\
\hline \hline$\Delta m[10]$ & $0.8^{0}$ \\
$\tau_{s}[9]$ & $0.03^{0}$ \\
\hline \hline
\end{tabular}

Table 2: Contributions to the systematic error on $\phi_{00}$. The systematic error from our measurement of $\phi_{00}$ is summarized separately ("Total") from the external error sources $\Delta m$ and $\tau_{S}$.

using the decay-time resolution function as extracted from the simulation. Any variation of the resolution function so that the resulting decay curve stays, within statistics, in agreement with the observed one, leads to a systematic shift in the value of $\phi_{00}$ of $\leq 0.6^{\circ}$. A possible variation in the decay-time resolution as a function of the decay-time has been studied by simulation. No evidence for such a dependence has been found and corresponding variations in the sensitive region within the statistical limits have a negligible effect on $\phi_{00}$.

For a small number of events, the reconstruction of the $\overline{\mathrm{K}}^{0}\left(\mathrm{~K}^{0}\right)$ decay-time is affected by the detection of a photon, which does not originate from the decay $\overline{\mathrm{K}}^{0}\left(\mathrm{~K}^{0}\right) \rightarrow 2 \pi^{0}$, but from the strong interaction or the decay of the accompanying charged particles in the calorimeter. Events with such photons contribute about $0.5 \%$ to the data according to simulations. In order to account for uncertainties in the simulation of such secondary photons, the number of these events has been varied within $50 \%$. We take the corresponding shift of $1.0^{\circ}$ as a contribution to the systematic error on $\phi_{00}$.

Uncertainties in the simulation of the residual background and in the determination of the pionic annihilation background from the experimental energy loss distributions in the inner scintillators are accounted for by allowing a variation of the individual background contributions within $50 \%$ as compared to the mean values of Table (1). This results in an error on $\phi_{00}$ of $0.5^{\circ}$, which is mainly due to the pionic annihilation background in the interference region.

Systematic uncertainties introduced within each $\overline{\mathrm{K}}^{0}\left(\mathrm{~K}^{0}\right)$ momentum subsample by a possible deviation of the $\xi$-values from a constant, and by a varying decay-time acceptance, have been studied. This adds less than $0.5^{\circ}$ to the systematic error on $\phi_{00}$.

The effect of coherent regeneration on the $\overline{\mathrm{K}}^{0}$ and $\mathrm{K}^{0}$ rates is taken into account when fitting the asymmetry. The systematic error on $\phi_{00}$, due to uncertainties in the regeneration amplitudes, amounts to $0.6^{\circ}$. These uncertainties arise from the fact that calculated values for the regeneration amplitudes [11] with limited precision had to be 
used [2]. Incoherent regeneration can be neglected.

The dependence of $\phi_{00}$ on $\Delta m$ (in units of $10^{7} \hbar \mathrm{s}^{-1}$ ) and $\tau_{S}$ (in units of ps) is given by:

$$
\phi_{00}=50.8^{\circ}+[0.26(\Delta m-527.4)]^{\circ}+\left[0.23\left(\tau_{S}-89.26\right)\right]^{\circ} .
$$

The error on $\Delta m$ using the value measured by CPLEAR [10] translates into an uncertainty on $\phi_{00}$ of $0.8^{\circ}$. The error on $\tau_{S}$ taken from the Particle Data Group [9] introduces a negligible error on $\phi_{00}$.

The contributions to systematic uncertainties in the determination of $\phi_{00}$ are summarized in Table (2).

\section{$6 \quad$ Summary and Conclusions}

$\mathrm{CP}$ violation has been observed for the first time in an experiment in which the initial neutral kaon is identified as being a $\overline{\mathrm{K}}^{0}$ or a $\mathrm{K}^{0}$, and decaying into two neutral pions. The measured particle-antiparticle asymmetry is a sign of $\mathrm{CP}$ violation independent of phenomenological descriptions. A technique of analysis has been developed for selecting the events for which the decay length of the neutral kaon could be reconstructed unambiguously and with sufficient accuracy. The detailed study of systematic uncertainties shows that the experimental method allows the measurement of $\phi_{00}$ with an accuracy of the order of $1.5^{\circ}$, given a sufficient number of events.

\section{Acknowledgements}

We would like to thank the CERN LEAR staff for their support and co-operation as well as the technical and engineering staff of our institutes. This work was supported by the following institutions: the French CNRS/Institut National de Physique Nucléaire et de Physique des Particules, the French Commissariat à l'Energie Atomique, the Greek General Secretariat of Research and Technology, the Netherlands Foundation for Fundamental Research on Matter (FOM), the Portugese JNICT and INIC, the Ministry of Science and Technology of the Republic of Slovenia, the Swedish Natural Science Research Council, the Swiss National Science Foundation, the UK Particle Physics and Astronomy Research Council (PPARC), and the US National Science Foundation.

\section{References}

[1] R. Adler et al., Phys. Lett. B286 (1992) 180.

[2] R. Adler et al., Measurement of the CP Violation Parameter $\eta_{+-}$using tagged $\mathrm{K}^{0}$ and $\overline{\mathrm{K}}^{0}$, CERN-PPE/95-107, submitted to Phys. Lett. B.

[3] For reviews, see for example K. Kleinknecht, Ann. Rev. Nucl. Science 26 (1976) 1; J. Steinberger, Proceedings of "CP Violation in Particle Physics and Astrophysics", Blois 1989, Ed. Frontieres.

[4] R. Adler et al., The CPLEAR detector at CERN, in preparation.

[5] R. Adler et al., Nucl. Instrum. and Methods, Phys. Res. A321 (1992) 458.

[6] B. Pagels, O. Behnke and H.-J. Gerber, Reconstruction of a Particle Decay containing intermediate Decays of invisible Neutrals, ETHZ-IPP Internal Report RP 95-04.

[7] B. Pagels, Ph.D. Thesis, University of Basle, 1992.

[8] See for example H. Wind, Principal Component Analysis and its Applications to Track Finding, in "Formulae and Methods in Experimental Data Evaluation", Vol. 3, CERN 1984.

[9] Particle Data Group, Phys. Rev. D50 (1994) 1173. 
[10] R. Adler et al., Measurement of $\mathrm{K}_{\mathrm{L}}^{0}-\mathrm{K}_{\mathrm{S}}^{0}$ mass difference using semileptonic decays of tagged neutral kaons, CERN-PPE/95-103, submitted to Phys. Lett. B.

[11] P.H. Eberhard, F. Uchiyama, Nucl. Instrum. and Methods, Phys. Res. A350 (1994) 144. 


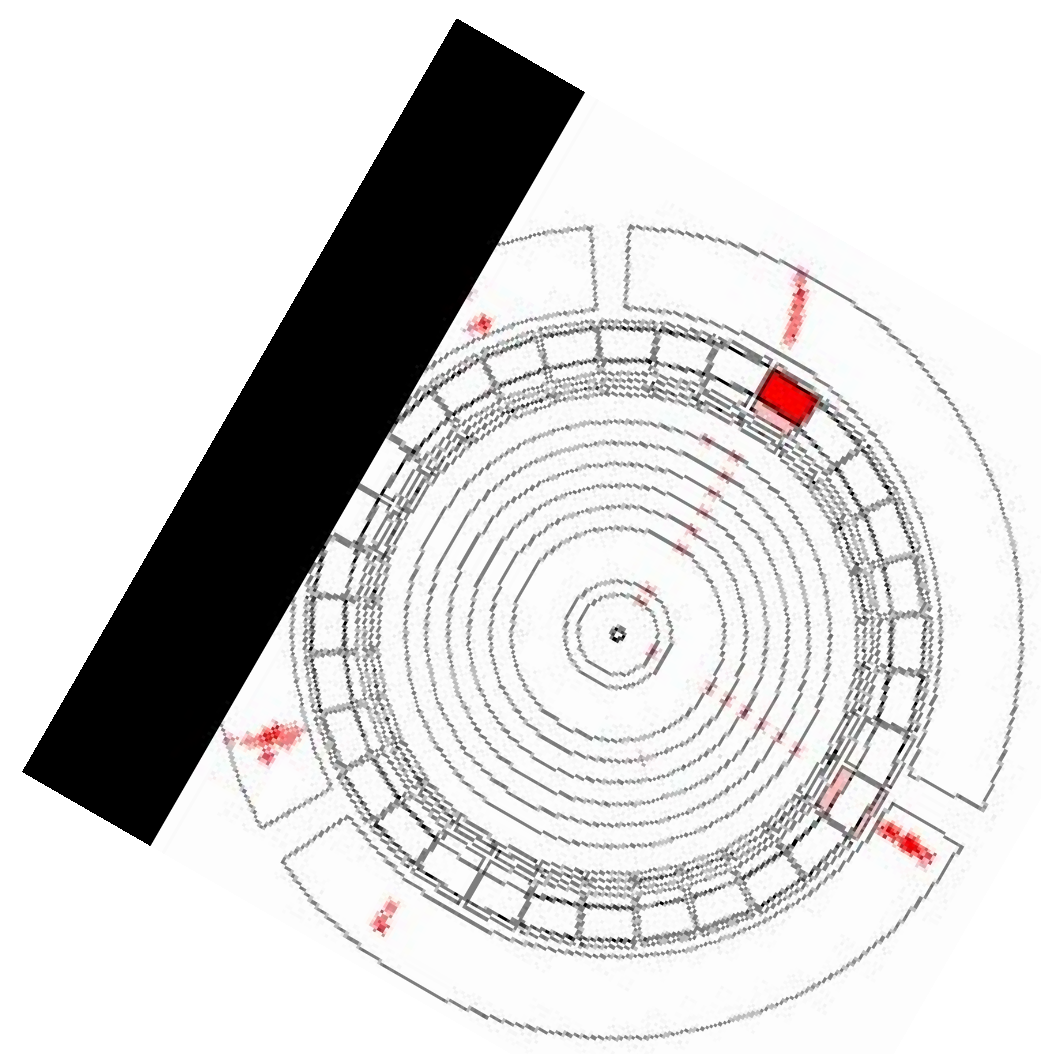

